\title{
Possible Experiment for the Demonstration of Neutron Waves Interaction with Spatially Oscillating Potential
}

\author{
Mădălina Mihaela Miloi ${ }^{1,2, *}$, Semyon Goryunov ${ }^{2}$, and German Kulin ${ }^{2}$ \\ ${ }^{1}$ Faculty of Physics, University of Bucharest, Bucharest, Romania \\ ${ }^{2}$ Joint Institute for Nuclear Research, Frank Laboratory of Neutron Physics, Dubna, Russia
}

\begin{abstract}
A wide range of problems in neutron optics is well described by a theory based on application of the effective potential model. It was assumed that the concept of the effective potential in neutron optics have a limited region of validity and ceases to be correct in the case of the giant acceleration of a matter. To test this hypothesis a new Ultra Cold neutron experiment for the observation neutron interaction with potential structure oscillating in space was proposed. The report is focused on the model calculations of the topography of sample surface that oscillate in space. These calculations are necessary to find an optimal parameters and geometry of the planned experiment.
\end{abstract}

\section{Introduction}

The beginning on the study of the interaction of neutrons with a moving matter was laid by the work [1]. Authors drew attention to the fact that the translational motion of the sample should lead to a change in the phase of the transmitted wave and it can take place only due to the movement of boundaries of matter. Later in experiments [2,3] the existence of an effect was observed when the sample moved along the direction of the neutron pulse and its absence when the sample moved parallel to its surface (moves inside stationary boundaries) [4].

The case of arbitrary motion of the sample remained for a long time practically unexplored. The question of the passage of neutrons through a material layer moving with acceleration was theoretically considered in [5-7] and it was concluded that energy of neutrons after passing it should be changed. The phenomenon of the change in the energy of neutrons passing through an accelerating matter was observed in the experiment with ultra-cold neutrons (UCN) $[8,9]$.

In $[10,11]$, suggestions were made about a possible violation of the dispersion law in an accelerating substance. The critical acceleration at which a significant deviation from dispersion law can be expected was estimated in [12]. This critical acceleration is proportional to the energy of neutrons and for UCN an estimate of the critical acceleration gives $4 \times 10^{7} \mathrm{~cm} / \mathrm{sec}^{2}[12]$. This acceleration is achievable at laboratory experiments. The

${ }^{*}$ Corresponding author: madalina.mihaela.miloi@gmail.com 
experimental approach for the observation of interaction of UCN with matter moving with giant acceleration was proposed in [13].

\section{The possible realization of the experiment}

In work [13] was proposed to compare the results of UCN passing through the NIF oscillating in space, with the results of the calculation in assumption that the potential model is valid for this system. It is planned to carry out measurements, for relatively low acceleration, which is lower than critical value, and for the acceleration of matter significantly exceeding the critical one.

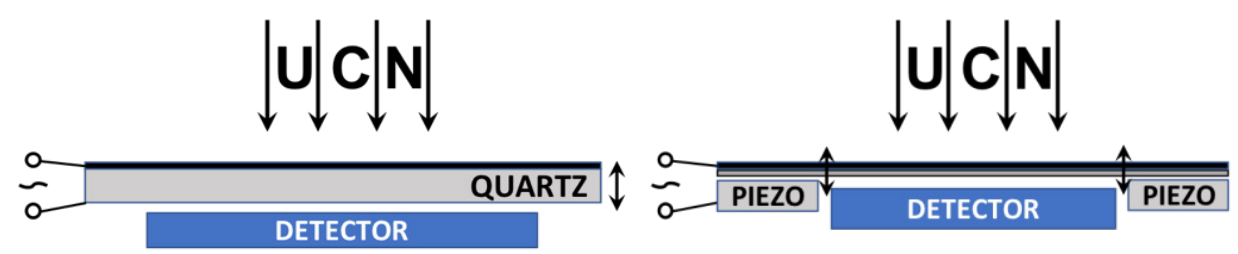

Fig. 1. Experimental scheme [13]. On the left side: a piezo-driver combined with NIF wafer, high frequency regime. On the right side: low frequency regime with a thick piezo.

In high frequency regime it is proposed to depose NIF directly to the quartz plate which serves as resonant piezo driver [13] (see fig. 1, left). The thickness of the plate must be equal or multiple to the half of ultrasonic wavelength. In the case of low frequency about $100 \mathrm{kHz}$ it is not possible to use the same configuration as for high frequency. In this case quartz plate should be ten times thicker that will lead to serious losses of UCN due to absorption in it. As possible solution, it was proposed [13] to use NIF deposed to the thin wafer which is fixed at the annular Piezo driver (see fig. 1, right).

\section{Equations and mathematics}

It is important that the surface of the sample is oscillated at frequencies from several $\mathrm{kHz}$ to several $\mathrm{MHz}$ as a whole, together with its boundaries or without significant deformation. The aim of this work was to find optimal geometric parameters of system at which the surface deformation would remain within $10 \%$. The difficulty lies in the fact that the thickness and material of the wafer have to be such that it remains sufficiently transparent for UCN.

Different configurations for the wafer were studied. All the simulations were done in ANSYS software. In simulations, the oscillation is represented by a sinusoidal timedependent displacement that is applied to the boundaries of the wafer. The rest of the disk is free to move following the inertial laws. Hence, a relative deformation could be defined in percents as

$$
D_{\text {rel }}=\left(A_{\max }-A\right) / A * 100
$$

where $A$ is the displacement amplitude characterizing the forcing load. $A_{\max }$ the maximum deformation on axis that is identical to the direction perpendicular to the plane of the disk that could appear because of the inertia of the disk, under the acceleration. The amplitude of oscillations was fixed and was about $14 \mu \mathrm{m}$. 
At figure 2 (left) results of simulations for plain disk of $12 \mathrm{~mm}$ in diameter for different thickness are shown. The simulations that were done, the range of frequencies was varied from $1 \mathrm{kHz}$ to $0.1 \mathrm{MHz}$. As we can see, at this diameter of free area it is not possible to achieve $100 \mathrm{kHz}$ at reasonable values of relative deformation. At $2 \mathrm{~mm}$ of sample thick it is possible to reach $50 \mathrm{kHz}$ only, but transparency of the sample for UCN in this case will be very small. Optimal parameters for relative deformation up to $100 \mathrm{kHz}$ reachable for the diameter of the free area of $5 \mathrm{~mm}$ and thickness of $0.6 \mathrm{~mm}$ only (figure 2, right), but this geometry is impossible to use in the experiment due to very small size of the free area.

It was understood that the deformation is affected by two parameters. The first is inertia of the freely moving region inside the oscillating annular surface and the second is elastic bonds at the boundary of the free region. Moreover, the inertia of the free region is proportional to the mass and the elastic coupling is proportional to the cross-sectional area at the boundary of the free region and these two parameters act against each other.
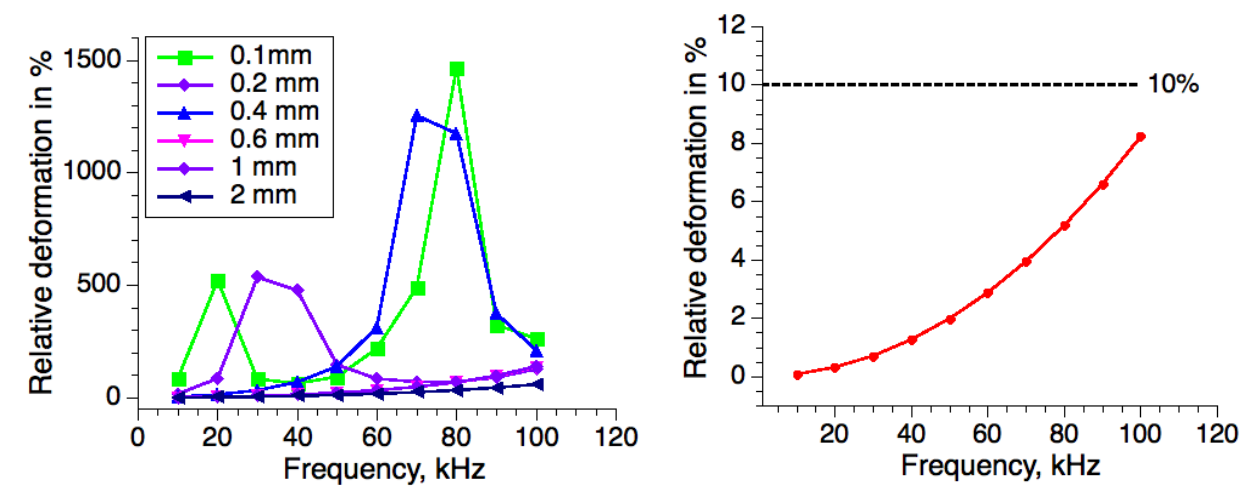

Fig. 2. The relative deformation dependence on frequency. On the left: for different thicknesses of the plain disk of $12 \mathrm{~mm}$ in diameter. On the right: for the plain disk of $5 \mathrm{~mm}$ in diameter and thickness 0.6 $\mathrm{mm}$.
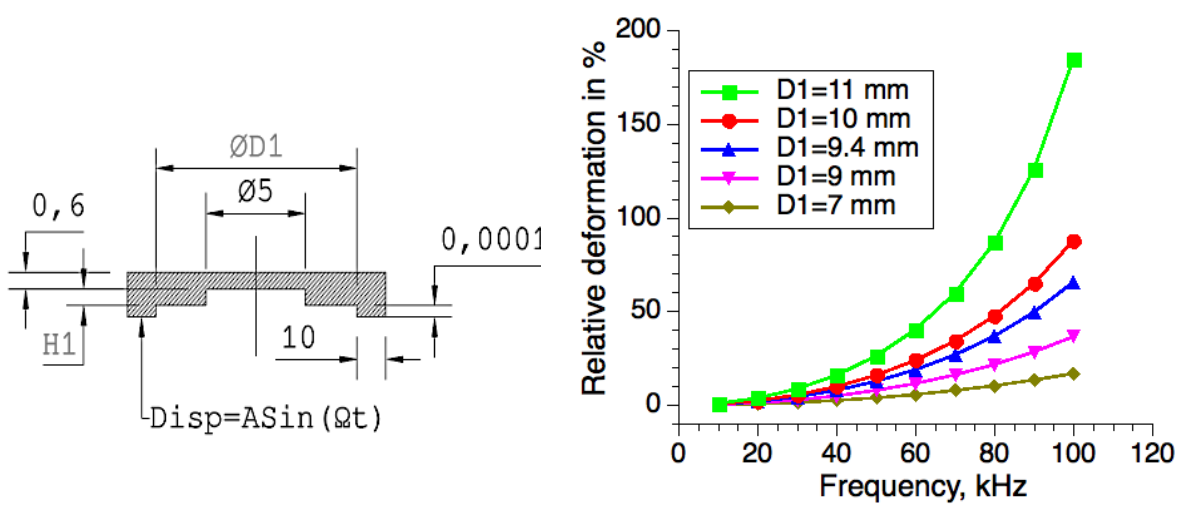

Fig. 3. The geometry of the disk with step-wise decreasing of the thickness. Dependence of the deformation on the frequency for different diameters D1 at a thickness of $\mathrm{H} 1=0.6 \mathrm{~mm}$.

Also, attempts were done to use step-wise decreasing of the thickness from the boundary of the free region to the center of the disk (figure 3) and to use the disk with inner part like a honeycomb grating. It allowed to reduce the mass of the free region without decreasing the cross section at the boundary of the free and oscillating region. Unfortunately, these attempts did not lead to any profit in comparing with plain disk geometry. 


\section{Conclusions}

A wide range of problems in neutron optics is well described by a theory based on application of the effective potential model. It was supposed that the concept of the effective potential in neutron optics have a limited region of validity and ceases to be correct in the case of the giant acceleration of a matter.

To test this hypothesis a new Ultra Cold neutron experiment for the observation neutron interaction with potential structure oscillating in space was proposed. For the implementation of the experiment, it is important that the surface of the sample is oscillated at frequencies from several $\mathrm{kHz}$ to several $\mathrm{MHz}$ as a whole, together with its boundaries or without significant deformation.

The model calculations of the topography of sample surface that oscillate in space were shown that, at frequencies of the order of $100 \mathrm{kHz}$ a relative deformation about $10 \%$ is impossible to be obtained for large size of the sample free area. It means that experimental approach itself should be seriously reconsidered.

Authors would like to thank A. I. Frank for permanent support and valuable suggestions.

\section{References}

1. M. A. Horn and A. Zeilinger, Clarendon Press, Oxford, 350 (1979)

2. A. G. Klein, G. I.Opat, A. Cimmino, et al., Phys. Rev. Lett. 46, 1551 (1981)

3. U. Bonse and A. Rumpf, Phys.Rev. Lett. 56, 2411 (1986)

4. M. Arif, H. Kaiser, S. A. Werner, et al., Phys. Rev. A 31, 1203 (1985)

5. V. I. Mikerov, PhD thesis The P.N. Lebedev Physical Institute, Moscow, 105 (1977)

6. F. V. Kowalski, Phys. Lett. A 182, 335 (1993)

7. V. G.Nosov, A. I. Frank, Phys. At. Nucl. 61, 613 (1998)

8. Frank A. I., Geltenbort P., Kulin G. V., Kustov D. V., Nosov V. G. and Strepetov A. N., JETP Letters 84, 363 (2006)

9. A. I. Frank, P. Geltenbort, P. Geltenbort, D. V. Kustov, G. V. Kulin, A. N. Strepetov, JETP Letters, 93:7 (2011)

10. A. I. Frank, P. Geltenbort, G. V. Kulin, D. V. Kustov, V. G. Nosov and A. N. Strepetov, Phys. At. Nucl. 71, 1656 (2008)

11. Frank A. I., Phys. Usp. 52, 397 (2009)

12. Frank A. I., JETP Lett. 100 (10), 613 (2015)

13. A.I. Frank, D.V. Kustov, G.V. Kulin, S.V. Goryunov, D.V. Roshchupkin, D.V. Irzhak, J. of Phys. Conf. Series 746(1), 012054(2016)

14. I. V. Bondarenko, V. I. Bodnarchuk, S. N. Balashov et al., Physics of Atomic Nuclei 62, 721 (1999) 